\title{
Biología reproductiva de ChUQUiraga AVELLANEDAE (Asteraceae), un arbusto endémico de la Patagonia
}

\author{
Reproductive biology of Chuquiraga aVellanedaE (Asteraceae), AN \\ ENDEMIC SHRUB OF PATAGONIA
}

Facundo T. Zaffaroni ${ }^{1 *} \mathbb{D}$, M. Victoria Campanella ${ }^{\mathbb{D}}$ y Fernando J. Martínez ${ }^{1,2} \mathbb{D}$

1. Facultad de Ciencias Naturales y de la Salud, Universidad Nacional de la Patagonia San Juan Bosco UNPSJB, Puerto Madryn, Chubut, Argentina.

2. Instituto Patagónico para

el Estudio de los Ecosistemas

Continentales, IPEEC-CONICET,

Puerto Madryn, Chubut, Argentina.

*facuzaffa@gmail.com

Citar este artículo

ZAFFARONI, F. T., M. V. CAMPANELLA Y F. J. MARTÍNEZ. 2021. Biología reproductiva de Chuquiraga avellanedae (Asteraceae), un arbusto endémico de la Patagonia. Bol. Soc. Argent. Bot. 56: 489-496.

DOI: https://doi. org/10.31055/1851.2372.v56. n4.33707

Recibido: 29 Jun 2021

Aceptado: 13 Sep 2021

Publicado en línea: 4 Nov 2021

Publicado impreso: 20 Dic 2021

Editor: Luz Allende iD

ISSN versión impresa 0373-580X

ISSN versión on-line 1851-2372

\section{SUMMARY}

Background and aims: Chuquiraga avellanedae (Asteraceae) is an endemic species of the Patagonian steppes and a key component of the ecosystem. Knowing the reproductive system and the dependence on pollinating agents is important for understanding gene flow and seed production. The objectives were to determine whether this species is self-incompatible and ambophilous.

M\&M: A field experiment was carried out and the following treatments were applied: cross-pollination, self-pollination, wind pollination, spontaneous pollination and control. Pollinating insects were recorded during two seasons.

Results: Seed production was significantly higher in the cross-pollination treatment and the control, differing from self-pollination and wind pollination. There was no seed production in the spontaneous pollination treatment. Flowers were frequently visited by insects mainly belonging to Hymenoptera and Diptera. There was interannual variation in the abundance of the major pollinator taxa.

Conclusions: Chuquiraga avellanedae is considered a self-incompatible species and a generalist-pollinated plant visited by many pollinators. This work reveals that pollinator insects are crucial for the reproductive success of this shrub. In agreement with previous knowledge, these findings indicate that the interaction $C$. avellanedae-insects represents a relevant component for ecosystem processes and services at regional scale.

\section{KEY WORDS}

Ambophily, breeding system, Chuquiraga avellanedae, manual pollination, pollinating insects, seed set.

\section{RESUMEN}

Introducción y objetivos: Chuquiraga avellanedae (Asteraceae) es una especie endémica de la estepa patagónica que representa un componente fundamental de los ecosistemas de la región. Conocer el sistema reproductivo, la dependencia a los agentes polinizadores y el ensamble de visitantes florales es importante para la comprensión de los patrones de flujo génico y la cantidad y la calidad de las semillas producidas. Los objetivos de este trabajo fueron determinar el grado de autoincompatibilidad de $C$. avellanedae y evaluar si es una especie ambófila.

M\&M: Mediante cruces controlados se puso a prueba la capacidad de producir semillas bajo distintos tratamientos: polinización cruzada, autopolinización forzada, polinización por viento, autopolinización espontánea y control. Se realizaron observaciones de los insectos polinizadores durante dos temporadas.

Resultados: La producción de semillas fue significativamente mayor en el tratamiento de polinización cruzada y el control, diferenciándose de la autopolinización forzada y la polinización por viento. No hubo producción de semillas en el tratamiento de autopolinización espontánea. Las flores fueron frecuentemente visitadas por especies de Hymenoptera y Diptera. Hubo variación inter-anual en la abundancia de los taxones más frecuentes.

Conclusiones: Chuquiraga avellanedae resultó autoincompatible y con un ensamble de polinizadores generalista. Los insectos polinizadores son cruciales para el éxito reproductivo de este arbusto. Coincidiendo con otros antecedentes, este trabajo indica que la interacción $C$. avellanedae-insectos representa un componente relevante en procesos y servicios ecosistémicos a nivel regional.

\section{Palabras claves}

Ambofilia, Chuquiraga avellanedae, insectos polinizadores, sistema reproductivo, polinización manual, producción de semillas. 


\section{INTRODUCCIÓN}

Estudios que aborden la reproducción de las plantas y su relación con los insectos polinizadores contribuyen a un mejor entendimiento del funcionamiento de los ecosistemas regionales ya que las interacciones plantapolinizadores son críticas no sólo para el flujo génico en la población de plantas (Hall et al., 1996), sino también para el mantenimiento de la biodiversidad y la integridad de los ecosistemas (Aizen \& Vázquez, 2006; Kearns \& Innouye, 1997). Además, esta información es básica para el desarrollo de estrategias de conservación tanto de la diversidad genética como taxonómica de una comunidad biológica (Tadey et al., 2009).

Chuquiraga avellanedae Lorentz (Asteraceae) es una especie endémica de la estepa patagónica y representa un componente fundamental en los ecosistemas terrestres de la región. Habita desde el sur de la provincia de Mendoza hasta el norte de Santa Cruz (Ezcurra, 1985). Es dominante en la Provincia Fitogeográfica Patagónica (Bisigato et al., 2016) y posee la capacidad de modelar el ambiente al fijar el suelo (Feijóo, 2006). Es utilizada como refugio, sitio de nidificación y alimentación de diversos taxones de artrópodos (Martínez et al., 2021) y posee propiedades de interés medicinal (Forcone, 2004; Tapparaci \& González, 2009). A pesar de la relevancia de esta especie arbustiva, se desconocen el grado de autoincompatibilidad, la dependencia a los agentes polinizadores y la composición del ensamble de visitantes florales.

Considerando que es un arbusto longevo con flores hermafroditas, y que una especie congénere $(C$. oppositifolia D. Don) es autoincompatible (Muñoz \& Arroyo, 2004), se espera que C. avellanedae presente autoincompatibilidad. Además, los caracteres florales de C. avellanedae (i.e. tamaño, color y oferta simultánea de flores) sugieren que el traslado del polen es realizado por insectos (Tadey et al., 2009; Torres \& Galetto, 2008). Por otro lado, el período de floración (Campanella \& Bertiller, 2008) coincide con los fuertes vientos del norte predominantes en el verano $(6 \mathrm{~m} / \mathrm{s}$ velocidad media máxima) (Palacio et al., 2014). Entonces, considerando que el viento es un componente característico en la Patagonia (Paruelo et al., 1998) y que la polinización por viento puede ser más importante en sitios abiertos que en los hábitats cerrados y boscosos (Medan \& Devoto, 2017), es probable que, además de los insectos, el viento tenga un papel importante en la polinización de $C$. avellanedae. Este fenómeno que presentan las especies vegetales polinizadas tanto por los insectos como por el viento se denomina ambofilia (Culley et al., 2002; Rech et al., 2016). Por ello, los objetivos de este estudio fueron determinar el grado de autoincompatibilidad de $C$. avellanedae y evaluar si es una especie ambófila.

\section{Materiales y Métodos}

\section{Área de estudio y especie focal}

El trabajo se realizó en la proximidad de la ciudad de Puerto Madryn, sitio ubicado en el ecotono entre las Provincias Fitogeográficas del Monte y Patagónica (-42,84 S; -65,02 O). En el ecotono coexisten comunidades vegetales, donde se destacan la de Larrea divaricada Cav. y Stipa spp., típica del Monte Austral y la de $C$. avellanedae, característica de la Provincia Fitogeográfica Patagónica (Bisigato et al., 2016). El clima es árido, templado y ventoso. La temperatura media anual es $13,5^{\circ} \mathrm{C}$ y la precipitación media anual es $233,8 \mathrm{~mm}$, con alta variación entre años y sin un patrón claro de estacionalidad (Casalini, 2016).

Chuquiraga avellanedae es un arbusto siempreverde de 0,5 a $1,5 \mathrm{~m}$ de altura de estructura ramosa y forma hemisférica. Las flores son tubulosas, amarillas y hermafroditas, se hallan reunidas de 8 a 15 en capítulos sésiles. Éstos se disponen de 1 a 3 en el extremo de los tallos o las axilas de las hojas. Las flores son amarillas, con papus pardusco tan largo como la corola. Es una especie protándrica (observación personal) y el polen tiene un diámetro de $30 \mu \mathrm{m}$ (Forcone, 2008). Su fruto es un aquenio, el cual contiene una sola semilla (Correa, 1971). El período de floración se extiende desde Noviembre hasta Marzo (Kröpfl et al., 2012).

\section{Tipo de sistema reproductivo y dependencia a los agentes polinizadores}

Con el fin de caracterizar el sistema reproductivo de $C$. avellanedae se realizó un experimento de cruces manuales. Se seleccionaron 30 individuos de C. avellanedae al comienzo del período reproductivo (Noviembre - Diciembre 2018). En cada arbusto se escogieron 5 ramas al azar y se retiraron algunos capítulos hasta alcanzar un único capítulo por rama. A cada rama se le asignó uno de los siguientes 


\section{F. T. Zaffaroni et al. - Biología reproductiva de Chuquiraga avellanedae}

tratamientos $(\mathrm{n}=30$ capítulos por tratamiento) (Tadey, 2007): 1) autopolinización forzada: flores embolsadas en pimpollo con friselina (excluye al polen) y polinizadas manualmente con polen de la misma planta, 2) autopolinización espontánea: flores embolsadas en pimpollo con friselina y sin intervención manual, 3) polinización cruzada: flores embolsadas en pimpollo con friselina y polinizadas manualmente con una mezcla de polen con-específico proveniente de otras plantas, 4) polinización por viento: flores embolsadas en pimpollo con voile de $0,2 \mathrm{~mm}$ de apertura de malla (excluye a los insectos y permite el paso del polen bajo la acción del viento) y sin intervención manual y 5) control: se dejaron flores sin embolsar expuestas a los agentes polinizadores naturales y sin intervención manual.

La intervención manual se realizó cuando los estigmas se encontraron abiertos (Kearns \& Innouye, 1993). La superficie de los estigmas se cubrió de polen pincelando suavemente con las anteras (Muñoz \& Arroyo, 2006). En cada capítulo se realizaron dos intervenciones manuales, la primera cuando se observó cuatro flores del capítulo con los estigmas bifurcados y la segunda luego de dos días posteriores a la primera intervención. Realizados los cruces, las flores fueron monitoreadas hasta la formación de los aquenios. En el caso del tratamiento control, las flores se embolsaron con friselina una vez pasado el período de receptividad de los estigmas florales para evitar pérdidas por dispersión. Los capítulos fueron colectados en Marzo de 2019, donde se contabilizó la cantidad de flores y de aquenios por capítulo. Además, se calculó el índice de auto-compatibilidad (ISI), que resulta de la razón entre la producción de frutos por autopolinización forzada (tratamiento 1) y la producción de frutos por polinización cruzada (tratamiento 3). Este índice va de 0 (totalmente autoincompatible) a 1 (autocompatible). Se considera a la planta como autoincompatible con valores del ISI por debajo de 0,2 (Zapata \& Arroyo, 1978).

\section{Ensamble de insectos polinizadores}

Para describir el ensamble de insectos polinizadores se realizaron observaciones directas de los insectos que visitaron a individuos de $C$. avellanedae durante el período de floración (Diciembre - Febrero) en las temporadas 2017/18 y 2018/19. Los muestreos se realizaron en intervalos de 10 min por planta (Muñoz \& Arroyo, 2004) en el período de mayor actividad de los insectos, entre las 11:00 y 14:00 hs (Tadey et al.,
2009). Durante ambas temporadas se realizaron 109 períodos de observación que equivalen a un total de 18,2 horas de observación directa. Se consideraron como polinizadores a aquellos insectos que tuvieron contacto con los órganos sexuales de las flores. Se registró a cada individuo y la cantidad de capítulos visitados. Además, cuando fue factible, se realizó la captura manual de los polinizadores. Los ejemplares fueron determinados al nivel taxonómico más bajo posible por medio de bibliografía especializada (Nieves-Aldrey et al., 2006; Pyrcz et al., 2016; Triplehorn et al., 2005). Finalmente, se estimó la abundancia relativa y la frecuencia de visitas de los distintos taxones que componen el ensamble de polinizadores de C. avellanedae. Los insectos fueron depositados en la colección entomológica del Instituto Patagónico para el Estudio de los Ecosistemas Continentales (IPEEC-CONICET).

\section{Análisis estadísticos}

Para evaluar la variación entre los efectos de los tratamientos se utilizaron modelos lineales generalizados mixtos (GLMM). Se consideró una variable respuesta binomial, con el número de aquenios producidos como "número de éxitos" y el número de flores vacías como "número de fracasos". Se incluyó a los tipos de cruzamiento como efecto fijo y a los individuos de $C$. avellanedae como efecto aleatorio (bloques), controlando de esta manera la variabilidad asociada a cada individuo al que se le aplicaron los tratamientos (Zuur et al., 2009). Se utilizó una distribución de error binomial (función link $=$ logit) y la validación del modelo se realizó a través de la inspección de los gráficos de residuales. Finalmente, se hicieron contrastes de Tukey (a posteriori) para analizar la variación entre tratamientos. Los análisis se realizaron utilizando los paquetes lme4 (Bates et al., 2015), multcomp (Hothorn et al., 2008) y DHARMa (Hartig, 2020) del software R (R Development Core Team, 2020). La rutina del análisis estadístico se encuentra en el material suplementario.

\section{Resultados}

Tipo de sistema reproductivo y dependencia a los agentes polinizadores

La cantidad de semillas producidas por capítulo varió entre los tratamientos $(\chi 2=43,345 ; p<0,0001$; g.l.=3; Tabla A, material suplementario). En el 
tratamiento de autopolinización espontánea $(n=27)$ no hubo producción de semillas, mientras que para la autopolinización forzada $(\mathrm{n}=26)$ y la polinización por viento $(\mathrm{n}=28)$ se obtuvieron valores bajos (Fig. 1). La cantidad de semillas producidas por capítulo fue significativamente mayor en los tratamientos control $(\mathrm{n}=30)$ y de polinización cruzada $(\mathrm{n}=27)$ (Fig. 1; Tabla $\mathrm{B}$, material suplementario). El número de flores por capítulo fue de 9,78 $\pm 0,13$ (media y error estándar).
El índice de auto-compatibilidad ISI fue de 0,14 , por lo cual el sistema reproductivo de $C$. avellanedae se clasificó como autoincompatible.

\section{Ensamble de insectos polinizadores}

El ensamble estuvo compuesto por 49 morfoespecies/especies pertenecientes a seis ordenes (Tabla 1). Hymenoptera y Diptera fueron los ordenes que presentaron la mayor riqueza relativa

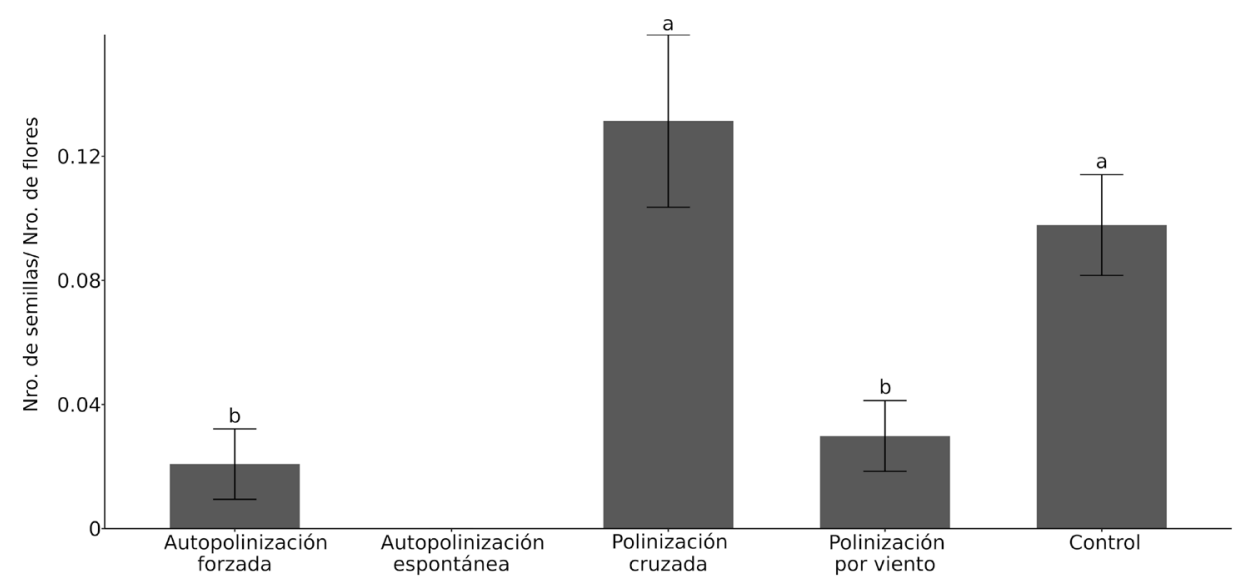

Fig. 1. Media y error estándar del número de semillas por flores del capítulo producidas en cada tratamiento. Letras distintas indican variación entre los tipos de cruzamiento.

Tabla 1. Polinizadores registrados durante los períodos de observación directa ( $N=578)$. En negrita se indican las siete morfo-especies/especies más frecuentes. En la categoria "otros" se agruparon los polinizadores que presentaron menos del $1 \%$ del total de las visitas. Las temporadas en la que no hubo registros se simboliza con -

\begin{tabular}{|c|c|c|c|c|}
\hline \multirow{2}{*}{ Orden } & \multirow{2}{*}{ Familia } & \multirow{2}{*}{ Morfo-especie/especie } & \multicolumn{2}{|c|}{ Frecuencia relativa (\%) } \\
\hline & & & $2017 / 18$ & $2018 / 19$ \\
\hline \multirow[t]{3}{*}{ Coleoptera } & Chrysomelidae & Chrysomelidae sp1 & 6,1 & 3,7 \\
\hline & Tenebrionidae & Tenebrionidae sp1 & - & 3,7 \\
\hline & Otros & +5 morfo-especies & 0,9 & 1,5 \\
\hline \multirow[t]{5}{*}{ Diptera } & Bombyliidae & Bombyliidae sp4 & 21,9 & 4,5 \\
\hline & Bombyliidae & Bombyliidae sp5 & 6,1 & 1,1 \\
\hline & Bombyliidae & Thyridanthrax sp & 6,1 & 6,5 \\
\hline & Tephritidae & Neosphaeniscus m-nigrum & 1,8 & 2,2 \\
\hline & Otros & +11 morfo-especies & 5,3 & 3,7 \\
\hline \multirow[t]{2}{*}{ Hemiptera } & Lygaeidae & Lygaeus alboornatus & 1,8 & 0,6 \\
\hline & Pentatomidae & Chinavia sp & - & 0,2 \\
\hline \multirow[t]{2}{*}{ Hymenoptera } & Halictidae & Halictidae sp1 & 4,4 & 67,2 \\
\hline & Otros & +18 morfo-especies & 11,4 & 3,4 \\
\hline \multirow[t]{2}{*}{ Lepidoptera } & Nymphalidae & Argyrophorus chiliensis & 34,2 & 0,4 \\
\hline & Otros & +4 morfo-especies & - & 1,1 \\
\hline \multirow[t]{2}{*}{ Neuroptera } & Chrysopidae & Chrysopidae sp1 & - & 0,2 \\
\hline & Total & 49 especies & 100 & 100 \\
\hline
\end{tabular}




\section{F. T. Zaffaroni et al. - Biología reproductiva de Chuquiraga avellanedae}

de morfo-especies/especies y mayor registro de visitas (Tabla 1).

Durante las dos temporadas se registraron 578 visitas. Argyrophorus chiliensis Bryk (Lepidoptera: Nymphalidae) fue el polinizador más frecuente en la primera temporada, en cambio para la segunda temporada lo fue Halictidae sp1 (Tabla 1).

Entre los taxones más frecuentes, la cantidad promedio de capítulos visitados por visita varió entre 1,46 y 3,42 correspondiendo a Chrysomelidae sp1 y Thyridanthrax sp (Diptera:
Bombyliidae) respectivamente. Chrysomelidae sp1 visitó en promedio 1,46 capítulos por visita (rango: 1-2 capítulos). Bombyliidae sp5 visitó en promedio 1,90 capítulos por visita (rango: 1-12). Halictidae sp1 visitó en promedio 2,03 capítulos por visita (rango: 1-13). Tenebrionidae sp1 visitó en promedio 2,24 capítulos por visita (rango: 1-2). A chiliensis visitó en promedio 2,95 capítulos por visita (rango: 1-12). Bombyliidae sp4 visitó en promedio 3,24 capítulos por visita (rango: 1-15). Finalmente, Thyridanthrax sp visitó un promedio de 3,42 capítulos por visita (rango: 1-14) (Fig. 2).

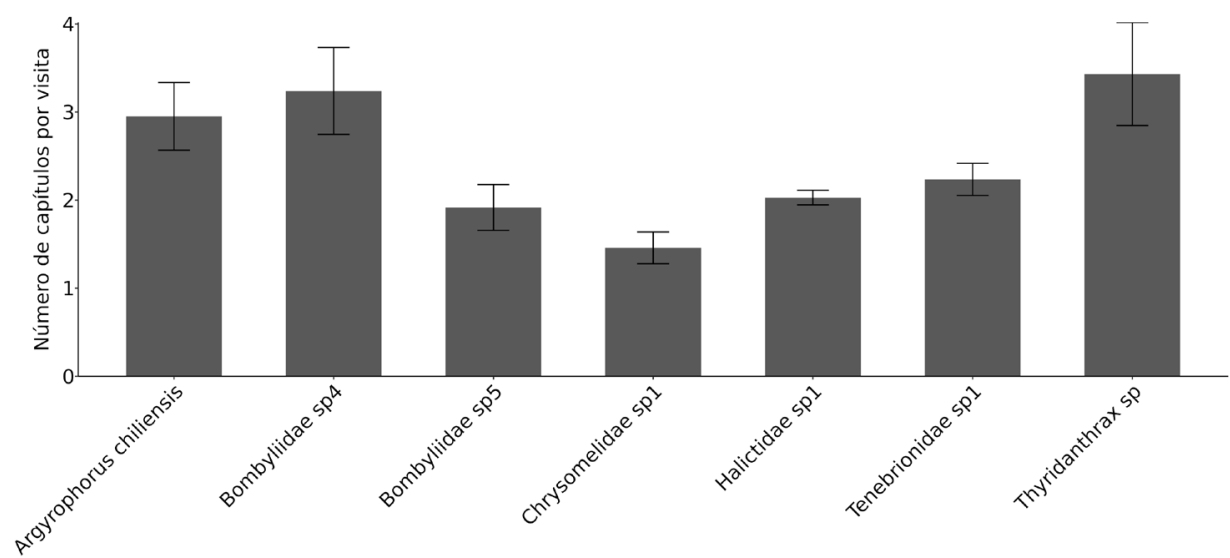

Fig. 2. Media y error estándar del número de capítulos visitados por visita de los siete taxones más frecuentes de polinizadores.

\section{Discusión}

Los resultados encontrados indican que $C$. avellanedae es autoincompatible. Por lo tanto, este arbusto necesita la presencia de otros individuos y de agentes polinizadores para reproducirse sexualmente de manera exitosa. Este resultado concuerda con el descripto para otra especie cercanamente emparentada al nivel taxonómico de género, $C$. oppositifolia, la cual es autoincompatible (Muñoz \& Arroyo, 2004). En este sentido, la polinización cruzada es considerada ventajosa respecto de la autofecundación porque produce una progenie genéticamente más variable y evita la depresión por endogamia, incrementando el potencial de adaptación ante eventuales cambios ambientales (Barrett, 2003).
En este trabajo se evidenció que la población de C. avellanedae estudiada depende principalmente de los insectos para reproducirse. En América del Sur se ha descripto polinización por insectos y colibríes para el género Chuquiraga (Abrahamczyk et al., 2017; Ezcurra, 2002). En consonancia con estos resultados, se describe que las especies del género que habitan en las Provincias Fitogeográficas del Monte y Patagónica son polinizadas por insectos (Ezcurra, 2002). Además, otros estudios mencionan que las especies de zonas áridas en Argentina también son polinizadas por insectos (Debandi et al., 2002; Tadey et al., 2009; Tadey, 2011). Con respecto a la ambofilia estos resultados sugieren que el viento no juega un rol importante como agente polinizador de $C$. avellanedae. Sin embargo, descartar la posibilidad de que presente ambofilia a lo largo de su rango de distribución (Mendoza - Santa Cruz) podría 
ser apresurado, ya que existen registros de variación entre polinización exclusiva por insectos y ambofilia para otro arbusto de Patagonia, Discaria chacaye (G. Don) Tortosa (Medan \& Devoto, 2017).

Las flores de C. avellanedae fueron visitadas por 49 morfo-especies pertenecientes a 6 ordenes de insectos, por lo que el ensamble de polinizadores se considera generalista. También, se observó variabilidad interanual en la composición específica y en la abundancia relativa entre los taxones que componen el ensamble. Estos resultados coinciden con lo encontrado en otros estudios (Chacoff et al., 2018; Vázquez \& Simberloff, 2004) donde se destaca que las interacciones son altamente dinámicas y varían mucho en el tiempo (Poisot et al., 2015). Frente a esta variabilidad temporal, la presencia de un ensamble generalista resultaría ventajosa. En este sentido, se ha encontrado que especies de polinizadores funcionalmente redundantes favorecen el éxito reproductivo de las plantas ya que, cuando alguno de los polinizadores está ausente o la abundancia es relativamente baja, la función como polinizador es reemplazada por otra especie del ensamble (Balvanera et al., 2005, 2006; Fenster et al., 2004; Morris, 2003).

Este trabajo aporta información novedosa sobre el sistema reproductivo y los visitantes florales de $C$. avellanedae, una especie endémica de la Patagonia y relevante en el ecosistema. Lo hallado indicaría que este arbusto es autoincompatible y depende principalmente de los insectos polinizadores para reproducirse de manera exitosa. También se encontró que $C$. avellanedae es una fuente de recursos para una elevada cantidad de insectos que visitan sus flores, $y$, por lo tanto, una especie vegetal relevante para los ensambles de polinizadores de la zona. Además, para la misma región, se ha encontrado que $C$. avellanedae es un hábitat importante para otros grupos funcionales de insectos (e.g. detritívoros y granívoros) (Dellape \& Cheli, 2007; Martínez et al., 2021) sugiriendo que la interacción $C$. avellanedae-insectos representa un componente relevante en procesos y servicios ecosistémicos claves como el ciclado de nutrientes y la polinización.

\section{Contribución de los aUtores}

Todos los autores han realizado conjuntamente y a partes iguales la colecta de datos, su interpretación y redacción del manuscrito.

\section{Agradecimientos}

Al Consejo Nacional de Investigaciones Científicas y Técnica (PIP 11220170100981 y PUEIPEEC-2016 22920160100044) y a la Universidad Nacional de la Patagonia San Juan Bosco (PI-1491) por proveer los fondos. A la Dirección de Flora y Fauna Silvestre de la Provincia de Chubut por los permisos de colecta (Disposición $\mathrm{N}^{\circ}$ 09/18). A la Dr. A. Zapata y al Dr. M. Devoto por la ayuda en la determinación de los especímenes.

\section{Bibliografía}

ABRAHAMCZYK, S., C. PORETSCHKIN \& S. S. RENNER. 2017. Evolutionary flexibility in five hummingbird/plant mutualistic systems: testing temporal and geographic matching. J Biogeogr. 44: 1847-1855.

AIZEN, M. A. \& D. P. VÁZQUEZ. 2006. Flower performance in human-altered habitats. In: HARDER, L. D. \& S. C. BARRETT (eds.), Ecology and evolution of flowers, pp.159-179. Oxford University Press.

BALVANERA, P., C. KREMEN \& M. MARTÍNEZRAMOS. 2005. Applying community structure analysis to ecosystem function: examples from pollination and carbon storage. Ecol. Applic. 15: 360-375. https://doi.org/10.1890/03-5192.

BALVANERA， P., A. B. PFISTERER, N. BUCHMANN, J. S. HE, T. NAKASHIZUKA, D. RAFFAELLI \& B. SCHMID. 2006. Quantifying the evidence for biodiversity effects on ecosystem functioning and services. Ecol. Letters 9: 1146-1156. https://doi.org/10.1111/j.1461-0248.2006.00963.x

BARRETT, S. C. 2003. Mating strategies in flowering plants: the outcrossing-selfing paradigm and beyond. Philos. Trans., Ser. B. 358: 991-1004. https://dx.doi.org/10.1098\%2Frstb.2003.1301

BATES, D., M. MÄCHLER, B. BOLKER \& S. WALKER. 2015. Fitting linear mixed efects models using lme4. J. Stat Softw. 67: 1-47. https://doi.org/10.18637/jss.v067.i01.

BISIGATO, A. J., L. A. HARDTKE, H. F. DEL VALLE, P. J. BOUZA \& R. G. PALACIO. 2016. Regional-scale vegetation heterogeneity in northeastern Patagonia: Environmental and spatial components. Community Ecol. 17: 8-16. https://doi.org/10.1556/168.2016.17.1.2 


\section{F. T. Zaffaroni et al. - Biología reproductiva de Chuquiraga avellanedae}

CAMPANELLA, M. V. \& M. B. BERTILLER. 2008. Plant phenology, leaf traits and leaf litterfall of contrasting life forms in the arid Patagonian Monte, Argentina. J. Veg. Sci. 19: 75-85.

https://doi.org/10.3170/2007-8-18333

CASAlini, A. I. 2016. Heterogeneidad de la vegetación en el ecotono entre las provincias fitogeográficas del monte y patagónica. Descripción y factores asociados. Tesis doctoral. Universidad Nacional del sur, Bahía Blanca, Buenos Aires, Argentina.

CHACOFF, N. P., J. RESASCO \& D. P. VÁZQUEZ. 2018. Interaction frequency, network position, and the temporal persistence of interactions in a plantpollinator network. Bull. Ecol. Soc. Amer. 99: 21-28. https://doi.org/10.1002/ecy.2063.

CORREA, M. N. 1971. Flora Patagónica. Ed. Colección Científica. Instituto Nacional de Tecnología Agropecuaria (INTA), Buenos Aires, Argentina.

CULLEY, T. M., S. G. WELLER \& A. K. SAKAI. 2002. The evolution of wind pollination in angiosperms. Trends Ecol. Evol. 17: 361-369. https://doi.org/10.1016/S0169-5347\%2802\%2902540-5

DEBANDI, G., B. ROSSI, J. ARANÍBAR, J. A. AMBROSETTI \& I. E. PERALTA. 2002. Breeding system of Bulnesia retama (Gillies ex Hook \&Arn.) Gris. (Zygophyllaceae) in the Central Monte Desert (Mendoza, Argentina). J. Arid Environm. 51: 141152. https://doi.org/10.1006/jare.2001.0924.

DELLAPE, P. M.\&G. H. CHELI. 2007. A new species of Anomaloptera Amyot \& Serville from Patagonia (Hemiptera: Lygaeoidea: Oxycarenidae). Zootaxa. 1528: 65-68. https://doi.org/10.11646/zootaxa.1528.1.4

EZCURRA, C. 1985. Revisión del género Chuquiraga (Compositae-Mutisieae). Darwiniana. 219-284.

EZCURRA, C. 2002. Phylogeny, Morphology, and Biogeography of Chuquiraga, an Andean-Patagonian Genus of Asteraceae-Barnadesioideae. Bot Rev. 68: 153-170.

FORCONE, A. 2004. Hierbas y arbustos frecuentes en el valle inferior del Río Chubut. Ed. UNS, Chubut, Argentina.

FORCONE, A. 2008. Pollen analysis of honey from Chubut (Argentinean Patagonia). Grana 47: 147158. https://doi.org/10.1080/10652460802106340.

FEIJÓO, M. S. 2006. Caracteres morfo anatómicos foliares comparados en tres especies Patagónicas del género Chuquiraga Juss. (Asteraceae). Naturalia Patagonica. 3: 11-28.
FENSTER, C. B., W. S. ARMBRUSTER, P. WILSON, M. R. DUDASH \& J. D. THOMSON. 2004. Pollination syndromes and floral specialization. Annual Rev. Ecol. Evol. Syst. 35: 375-403. https://doi.org/10.1146/annurev.ecolsys.34.011802.132347

HALL, P., S. WALKER \& K. BAWA. 1996. Effect of forest fragmentation on genetic diversity and mating system in a tropical tree, Pithecellobium elegans. Conservation Biol. 10: 757-768.

https://doi.org/10.1046/j.1523-1739.1996.10030757.x

HARTIG, F. 2020. DHARMa: residual diagnostics for hierarchical (multilevel/mixed) regression models. $\mathrm{R}$ package version 0.3.3.0. https://CRAN.R-project. org/package=DHARMa.

HOTHORN, T., F. BRETZ \& P. WESTFALL. 2008. Simultaneous inference in general parametric models. Biometr. J. 50: 346-363. https://doi.org/10.1002/bimj.200810425

KEARNS, C. A. \& D. W. INOUYE. 1993. Techniques for pollination biologists. University press of Colorado.

KEARNS, C. A. \& D. W. INOUYE. 1997. Pollinators, flowering plants, and conservation biology. Bioscience. 47: 297-307. https://doi.org/10.2307/1313191

KRÖPFL, A. I., N. M. VILLASUSO \& G. PETER. 2012. Guía para el reconocimiento de especies de los pastizales del Monte Oriental de Patagonia. Ed. INTA, Bariloche, Argentina.

MARTÍNEZ, F. J., P. M. DELLAPÉ, A. J. BISIGATO \& G. H. CHELI. 2021. Native shrubs and their importance for arthropod diversity in the southern Monte, Patagonia, Argentina. J. Insect Conserv. 25: 27-38.

http://dx.doi.org/10.1007/s10841-020-00283-7

MEDAN, D. \& M. DEVOTO. 2017. Ambophily, not entomophily: the reproduction of the perennial Discaria chacaye (Rhamnaceae: Colletieae) along a rainfall gradient in Patagonia, Argentina. Pl. Syst. Evol .303: 841-851. https://doi.org/10.1007/s00606-017-1417-8

MORRIS, W. F. 2003. Which mutualisms are most essential? Buffering of plant reproduction against the extinction of pollinators. In: T. MCPHEARSON $\mathrm{P} \&$ P.J. MORIN, The importance of species: perspectives on expendability and triage. Integr. Comp. Biol. 43(4): 260-280. https://doi: $10.1093 / \mathrm{icb} / 43.4 .603$

MUÑOZ, A. A. \& M. T. ARROYO. 2004. Negative impacts of a vertebrate predator on insect 
pollinator visitation and seed output in Chuquiraga oppositifolia, a high Andean shrub. Oecologia. 138: 66-73. https://doi.org/10.1007/s00442-003-1405-2

MUÑOZ, A. A. \& M. T. ARROYO. 2006. Pollen limitation and spatial variation of reproductive success in the insect-pollinated shrub Chuquiraga oppositifolia (Asteraceae) in the Chilean Andes. Arctic Antarc. Alpine Res. 38: 608-613. https://doi.org/10.1657/15230430(2006)38[608:PLASVO]2.0.CO;2

NIEVES-ALDREY, J. L., F. FONTAL-CAZALLA \& F. FERNÁNDEZ. 2006. Introducción a los Hymenoptera de la Región Neotropical. Universidad Nacional de Colombia.

PALACIO R. G., A. J. BISIGATO \& P. J. BOUZA. 2014. Soil erosion in three grazed plant communities in northeastern Patagonia. Land Degrad. Dev. 25: 594-603. https://doi.org/10.1002/ldr.2289

PARUELO, J. M., E. G. JOBBÁGY \& O. E. SALA. 1998. Biozones of patagonia (Argentina). Ecol. Austral. 8: 145-153.

POISOT, T., D. B. STOUFFER \& D. GRAVEL. 2015. Beyond species: why ecological interaction networks vary through space and time. Oikos. 124: 243-251. https://doi.org/10.1111/oik.01719

PYRCZ, T., A. UGARTE, P. BOYER, A. M. SHAPIRO \& D. BENYAMINI. 2016. An updated list of the butterflies of Chile (Lepidoptera, Papilionoidea and Hesperioidea) including distribution, flight period and conservation status: part II, Subfamily Satyrinae (Nymphalidae), with the descriptions of new taxa. Bol. Mus. Nac. Hist. Nat. Santiago de Chile. 65.

R DEVELOPMENT CORE TEAM. 2020. R: A language and environment for statistical computing. R Foundation for Statistical Computing, Vienna, Austria. Disponible en: https://www.R-project.org/

RECH, A. R., B. DALSGAARD, B. SANDEL, J. SONNE, J. C. SVENNING, N. HOLMES \& J. OLLERTON. 2016. The macroecology of animal versus wind pollination: ecological factors are more important than historical climate stability. Plant Ecol. Divers. 9: 253-262.

https://doi.org/10.1080/17550874.2016.1207722

TADEY, M. 2007. Efectos del pastoreo sobre la polinización y producción de semillas en el Monte del NO Patagónico. Tesis Doctoral, CRUB-UN del Comahue, Bariloche, Río Negro, Argentina.

TADEY, M., J. C. TADEY \& N. TADEY. 2009. Reproductive biology of five native plant species from the Monte Desert of Argentina. Bot. J. Linn. Soc. 161: 190-201. https://doi.org/10.1111/j.1095-8339.2009.01001.x

TADEY, M. 2011. Reproductive biology of Monttea aphylla (Scrophulariaceae). Austral. J. Bot. 59: 713718. http://dx.doi.org/10.1071/BT10282

TAPPARACI, O. F. \& C. C. GONZÁLEZ. 2009. Guía escolar para la identificación de animales y plantas comunes del noreste del Chubut. Ed. Fondo para las Américas-Asociación Ecológica de la Patagonia.

TORRES, C. \& L. GALETTO. 2008. Importancia de los polinizadores en la reproducción de Asteraceae de Argentina Central. Acta Bot. Venez. 31: 473-494.

TRIPLEHORN, C. A., N. F. JOHNSON \&D. J. BORROR. 2005. An introduction to the study of insects. 7th ed. Australia: Thomson, Brooks/Cole.

VÁZQUEZ, D. P. \& D. SIMBERLOFF. 2004. Indirect effects of an introduced ungulate on pollination and plant reproduction. Ecol. Monogr. 74: 281-308. https://doi.org/10.1890/02-4055

ZAPATA, T. R. \& M. T. K. ARROYO. 1978. Plant reproductive ecology of a secondary deciduous tropical forest in Venezuela. Biotropica. 10: 221230.

ZUUR, A., E. N. IENO, N. WALKER, A. A. SAVELIEV \& G. M. SMITH. 2009. Mixed effects models and extensions in ecology with R. Springer Science \& Business Media. 